Mots. Les langages du politique

\title{
De proximité. D’une parenté juridique au pragmatisme syndical
}

Josette Lefèvre et Maurice Tournier

\section{(2) OpenEdition \\ Journals}

Édition électronique

URL : https://journals.openedition.org/mots/249

DOI : $10.4000 /$ mots. 249

ISSN : 1960-6001

Éditeur

ENS Éditions

Édition imprimée

Date de publication : 1 mars 2005

Pagination : 145-150

ISBN : 2-84788-077-1

ISSN : 0243-6450

Référence électronique

Josette Lefèvre et Maurice Tournier, «De proximité. D'une parenté juridique au pragmatisme syndical », Mots. Les langages du politique [En ligne], 77 | 2005, mis en ligne le 31 janvier 2008, consulté le 23 avril 2022. URL : http://journals.openedition.org/mots/249; DOI : https://doi.org/10.4000/mots.249 


\section{DES MOTS EN POLITIQUE}

\section{De proximité, d'une parenté juridique au pragmatisme syndical}

Proximité fait partie de ces termes français de seconde génération qui ont été repuisés dans le latin classique, les mots de première génération venant, eux, directement du gallo-roman après usure médiévale de leur prononciation bas latine. C'est ainsi que l'étymon prope, près, se retrouve dans deux filières lexicales, dites «populaire» et "savante». Mais on peut être proche dans trois univers, l'espace, le temps, la parenté. Les valeurs spatiales et temporelles étaient surtout portées en latin par prope et son comparatif propior, propius, d'où sont sorties les séries populaires médiévales des pruef, prof, puis proche, prochet $\left(13^{\mathrm{e}}\right.$ siècle) et de dérivés (issus d'un possible *propeanus) : prucein, procein, proceain, prochien, prochain, tout proche. Exemples classiques :

Mes sœurs, j'entends du bruit dans la chambre prochaine. (Racine, Esther, 2, 8)

J'ai lu dans ses regards sa prochaine vengeance. (Racine, Mithridate, 4, 2)

La valeur de parenté a aussi existé dès le $12^{\mathrm{e}}$ siècle, dans les mots de cette filière (exemple : le prochain parent). Le Trésor de la langue française en cite plusieurs exemples. De même, à partir du superlatif proximus et de sa nominalisation proximitas, voisinage, l'ancien français a fait fructifier l'héritage populaire avec proisme, prisme et proismeté, spécialisés dans ces rapports de parenté. Déjà, chez César, proximi signifiait les «proches». D’où l'expression classique proximité de sang.

C'est cette valeur parentale qui se retrouve d'abord dans proximité, emprunt francisé de proximitas, dont l'usage, très spécifique, ne remonte pas audelà du $14^{\mathrm{e}}$ siècle. Il s'agit du "cas de proximité », qui figure dans la Somme rurale due au juriste Boutillier. Ce terme savant prendra le pas sur la lignée populaire précédente, tout en s'en inspirant : à propos d'héritages, on invoquait le «cas en droit de premesse», le proesme (ou presme en Bretagne et Béarn) étant «lignager ou parent, en la Coutume d'Anjou, du Maine et en l'ancienne de Boulenois», «quand l'héritage revient au plus prochain par

Université de Picardie, CURAPP-CNRS, josette.lefevre@u-picardie.fr 
ligne $»^{1}$. Et Ragueau d'ajouter : «Boutillier appelle Proisme le parent proche de sang du côté de pere et de mere». Les Coutumes de Beaumanoir parlaient au $13^{\mathrm{e}}$ siècle de «reson de lignage» et de «reson de prochaineté de lignage $»^{2}$. De proximité se substitue donc aux anciennes locutions de premesse et de prochaineté. Signe de relatinisation?

Cet exemple nous intéresse à plusieurs titres. La locution de proximité est nettement figée : elle figure sans changement dans le jargon juridique. Cas peut se trouver suivi par d'autres compléments : cas de présent (flagrant délit), cas de saisine, cas de nouvelleté (récidive), cas de mesaventure (malchance), cas de rompture, etc. Il peut aussi céder la place à quelques autres termes: privilège, droit, raison, matière, etc. Mais de proximité reste intangible, avec pour seule variante le pléonasme cas en matière de.

Ce cas de proximité qui s'installe dans la langue juridique n'a pourtant que peu à voir avec nos modernes commerce, police... de proximités. Certes, la grammaire traditionnelle pourrait parler de complément de nom pour les deux types d'expressions. Mais la fonction sémantique se démarque de cette analogie : dans cas de proximité, le second terme est, comme le dit Grevisse, dominant et le premier joue un rôle de simple qualifiant, la proximité (parentale) étant considérée comme catégorie particulière, alors que dans commerce de proximité, par exemple, c'est le premier terme qui est dominant et le second qui lui ajoute une qualification : le commerce est caractérisé par sa proximité (spatiale) vis-à-vis de sa clientèle. Il y a solution de continuité entre les deux types d'emploi. La locution figée déterminative de proximité à rôle adjectival serait donc une création moderne, et tellement récente, semble-t-il, qu'il faut attendre 1962 pour la voir apparaitre dans un dictionnaire (Trésor de la langue française).

Entretemps, la signification du mot proximité a élargi son champ d'application, recouvrant peu à peu toutes les valeurs originelles, en particulier spatiales : «De nos jours, conclut le Robert historique, le mot exprime essentiellement la notion spatiale de voisinage (1543). » Notion qu'illustre la nouvelle locution de proximité. Dans la citation du Trésor de la langue française, une «fusée de proximité» est une «fusée-détonateur, qui se déclenche dès qu'elle se trouve à proximité de l'objectif pour le détecter» ${ }^{4}$. Cet exemple technologique est suivi dans l'usage par quelques autres du même type. Vingt ans plus

1. Ainsi que l'explique François Ragueau dans son Indice des droits royaux et seigneuriaux de 1583, publié sous le titre Glossaire du Droit françois par Eusèbe de Laubrière (édition de 1704 reproduite par Slatkine reprints, 1969). Voir p. 395 et 393.

2. Exemples dans Philippe de Beaumanoir, 1970 [réédition], Coutumes de Beauvaisis, Paris, Picard, t. 2, p. 260. La graphie du mot varie d'un manuscrit à l'autre : prochaineté, proismeche, proismeté... (ibid., t. 1, p. 298).

3. Et élu de proximité : "Jouant les élus de proximité soucieux du confort de ses administrés, Philippe Douste-Blazy réclame donc que les avions [pour Toulouse] aillent atterrir et décoller ailleurs» (Libération, $1^{\text {er }}$ novembre 2004).

4. Trésor de la langue française, 1988, Paris, Gallimard, t. 13, p. 1408. 
tard, on en trouve dans le discours syndical l'emploi banalisé. Un rapprochement des premières occurrences apparues dans la parole officielle confédérale, que représentent les résolutions votées par les congrès nationaux des quatre centrales ouvrières représentatives, est suggestif de la pénétration progressive de la locution dans le milieu populaire sur une trentaine d'années (19722003)5. Examinons le tableau de ces occurrences.

\begin{tabular}{|c|c|c|c|c|}
\hline & Emplois de la locution & $1980-1989$ & 1990-1999 & $2000-2003$ \\
\hline $\begin{array}{l}\text { CFDT } \\
F=17^{6}, \\
\text { sur } \\
132006 \\
\text { occ. }\end{array}$ & $\begin{array}{l}\text { Médecine de proximité } \\
\text { Équipements de - } \\
\text { Syndicalisme de - } \\
\text { Services de - } \\
\text { Emplois de - } \\
\text { Lieux de - } \\
\text { Stratégie de - }\end{array}$ & $\begin{array}{l}1985 \text { (1 occ.) } \\
1985(2) \\
1988(3) \\
1988(1)\end{array}$ & $\begin{array}{l}1992(1), 1995(4) \\
1995(2)\end{array}$ & $\begin{array}{l}2002(1) \\
2002(1)\end{array}$ \\
\hline $\begin{array}{l}\text { CFTC } \\
F=20, \\
\text { sur } \\
175565 \\
\text { occ. }\end{array}$ & $\begin{array}{l}\text { Loisirs de proximité } \\
\text { Communication de - } \\
\text { Emplois de - } \\
\text { Soins de - } \\
\text { Syndicalisme de - } \\
\text { Relation de - } \\
\text { Activité de - } \\
\text { Protection de - } \\
\text { Solidarité de - } \\
\text { Solidarités de - } \\
\text { Commerces de - } \\
\text { Image de - } \\
\text { Services de - } \\
\text { Volonté de - }\end{array}$ & $1981(2)$ & $\begin{array}{l}1990(1) \\
1996(1) \\
1996(1) \\
1999(2) \\
1999(1) \\
1999(1) \\
1999(1) \\
1999(1) \\
1999(1) \\
1999(1) \\
1999(1) \\
1999(1) \\
1999(1)\end{array}$ & \\
\hline $\begin{array}{l}\text { CGT } \\
F=32, \\
\text { sur } \\
561225 \\
\text { occ. }\end{array}$ & $\begin{array}{l}\text { Structures de proximité } \\
\text { Équipements de - } \\
\text { Centres de - } \\
\text { Travail de - } \\
\text { Moyens de - } \\
\text { Syndicalisme de - } \\
\text { Besoins de - }\end{array}$ & & $\begin{array}{l}1992(1) \\
1995(1) \\
1995(1) \\
1995(1) \\
1999(1) \\
1999(5)\end{array}$ & $\begin{array}{l}2003(2) \\
2003(1)\end{array}$ \\
\hline $\begin{array}{l}F O \\
F=6, \\
\text { sur } \\
158397 \\
\text { occ. }\end{array}$ & $\begin{array}{l}\text { Information de proximité } \\
\text { Loisirs de - } \\
\text { Tissu hospitalier de - } \\
\text { Service hospitalier de - }\end{array}$ & & 1996 (1) & $\begin{array}{l}2000(1) \\
2000(1) \\
2000(1)\end{array}$ \\
\hline Total & & 9 occ. & 32 occ. & 8 occ. \\
\hline
\end{tabular}

5. Les congrès cédétistes avaient lieu tous les trois ans et, à partir de 1998, tous les quatre ans. Il en est de même à la CGT, officiellement à partir de 1999. La CFTC tenait son congrès national tous les deux ans et, à partir de 1981, tous les trois ans. FO tenait le sien tous les trois ans et, à partir de 1980, avec des intervalles variant entre trois et cinq ans.

6. F : il s'agit de la fréquence du mot proximité lui-même. 
On ne rencontre aucun exemple de la locution déterminative de proximité dans ce corpus de plus d'un million d'occurrences avant la date de 1981. Cette absence totale pendant les années soixante-dix ne veut pas dire qu'elle n'était pas en usage à l'époque. Certes, le bond réalisé pendant la seule décennie quatre-vingt-dix ( 32 occurrences contre 9 pendant les vingt premières années de notre corpus) semble aller dans le sens d'une amplification et d'un élargissement de l'usage, mais il ne conclut pas en faveur d'une absence au cours des années antérieures.

Signifie-t-il que la notion de "proximité» n'était pas encore entrée dans les priorités des revendications confédérales ? Non plus. Le mot lui-même et surtout la locution prépositive à proximité (de) apparaissent dès les années soixante-dix du corpus. En témoigne le tableau suivant :

\begin{tabular}{|l|l|l|l|}
\hline & \multicolumn{1}{|c|}{ à proximité de } & \multicolumn{1}{c|}{ la... proximité } & \multicolumn{1}{c|}{ de proximité } \\
\hline CFDT & 1 (1976) & & $16(1985-2003)$ \\
\hline CFTC & $1(1999)$ & $3(1996-1999)$ & $17(1981-1999)$ \\
\hline CGT & $15(1972-1992)$ & $4(1992-2003)$ & $13(1992-2003)$ \\
\hline FO & & $2(1980-2000)$ & $4(1996-2000)$ \\
\hline Total & 17 occ. & 9 occ. & 50 occ. \\
\hline
\end{tabular}

Il se produit même un phénomène de relais entre les deux expressions, dont la CGT porte le plus évident témoignage. Les compléments circonstanciels qui utilisent la locution prépositive, très présents en début de période, cèdent la place aux lexies qui, en milieu et fin de période, font appel au complément de nom. Mais ce relais grammatical, ce ralliement cégétiste à de proximité, accompagne une évolution des thèmes : c'est ainsi que les demandes «d'installation de salles de repos à proximité des lieux de travail», celles "d'installations (sportives) à l'intérieur ou à proximité immédiate de l'entreprise» et celles de «logement à proximité du lieu de travail» qui arrivent, nombreuses, entre 1972 et 1992, cèdent la place, à partir de 1992, à des réclamations d' « équipements (de soins) de proximité» de «structures», «centres» ou «moyens (sanitaires) de proximité» et surtout, à la proclamation d'un nécessaire «syndicalisme de proximité », «tenant compte des besoins de proximité syndicale» (2003). Cette mutation thématique n'est pas générale. À la CFDT, on parle dès 1988 de «syndicalisme de proximité ». Il semble que, sur ce point, la CFDT étant le lanceur du néologisme, il faille attendre dix ans (1999) pour que la lexie, suffisamment dédouanée de son origine, puisse être employée sans risquer l'accusation de suivisme. La maturation idéologique aurait, dans ce cas, précédé la mutation langagière... Car, chez les deux centrales, avec dix ans d'intervalle, il s'agit 
d'une mutation de slogan révélatrice du passage d'un «syndicalisme de masse et de classe» (CFDT) ou «de classe et de masse» (CGT) à un «syndicalisme d'adhérents » et «de proximité »7 (1999).

Ne faudrait-il pas aller plus loin dans les hypothèses explicatives ? On sait que les discours sociaux, en particulier le syndical et le politique, ne fonctionnent pas en vase clos mais en prise directe avec les usages ambiants. En France, 1981 a vu l'arrivée de la gauche au gouvernement; un discours de justification de la décentralisation ${ }^{8}$, accompagnant les lois-cadres Defferre, s'est peu à peu mis en place et Michel Rocard, dans son discours à l'Assemblée du 29 juin 1988, insiste, par exemple, sur une conception nouvelle de la police, qui «n'est pas seulement un instrument de prévention et de répression », mais aussi "un important service de proximité sociale » ${ }^{9}$. Des notions comme «le pays», «le terrain», «le local» reprennent de l'importance. Jacques Delors insistait en 1994, dans ses entretiens avec Dominique Wolton, sur «l'importance du local [...] pour nourrir le lien social, pour étayer la solidarité et, par voie de conséquence, pour créer des emplois qui correspondent à ce qu'on appelle aujourd'hui, puisque la formule est entrée dans le langage politique courant, des besoins de proximité $\aleph^{10}$. Ce discours correspondait à une logique de la construction européenne, en insistant sur les réalités régionales et locales et, comme le dit Jacques Delors, « la démocratie à portée de la main ${ }^{11}$. Les candidats à l'élection présidentielle de 1995 s'inscrivent dans ce langage. Lionel Jospin glose à son tour l'expression police de proximité par : "Je veux développer une police de proximité, accessible, efficace, au contact direct et permanent de la population. $»^{12}$

La CFDT participe la première à cette vague décentralisatrice par un discours où elle reprend des formules diffusées à l'époque. Apparaissent ainsi, aux congrès de 1985 et de 1988, les expressions médecine de proximité, services de proximité, etc., puis, dans les années quatre-vingt-dix, c'est le bond fréquentiel généralisé signalé plus haut. Du côté de la CGT, la nécessité de rejoindre le concert européen entraine la montée d'un discours de plus en plus

7. Voir A.-M. Hetzel, J. Lefèvre, R. Mouriaux, M. Tournier, 1998, Le syndicalisme à mots découverts. Dictionnaire des fréquences (1971-1990), Paris, Syllepse, p.318. Adhérents et proximité sont eux-mêmes proches dans les discours : N. Notat parle, à Montpellier (1995), d'un "syndicalisme d'adhérents, de pratiques participatives et de proximité».

8. À relents plus ou moins soixante-huitards. Aux «États généraux des pays» (1982), M. Rocard rappelle des slogans vieux de dix à quinze ans : «Voici maintenant une dizaine d'années que le mot d'ordre «Vivre, travailler, décider au pays» a fait irruption dans la vie politique [...]. II n’y avait rien là pour déplaire à celui qui, il y a plus de quinze ans, parlait déjà de décoloniser la province» (1986, À l'épreuve des faits. Textes politiques 1979-1985, Paris, Le Seuil, p. 183).

9. M. Rocard, 1989, Un pays comme le nôtre. Textes politiques 1986-1989, Paris, Le Seuil, p. 118.

10. J. Delors, 1994, L'unité d'un homme, Paris, Odile Jacob, p. 86-87.

11. Ibid., p. 383.

12. L. Jospin, 1995, 1995-200o. Propositions pour la France, Paris, Stock, p. 109. 
conforme aux exigences de la Confédération européenne des syndicats. L'apparition du figement de proximité pour qualifier l'adaptation de son syndicalisme correspond à cette période. II a le mérite, pour la CGT comme pour les autres confédérations, de répondre à des besoins proprement syndicaux de rapprochement avec les salariés et les «privés d'emploi ». On constate en effet que la multiplication des situations de précarité, l'éloignement des décideurs, la baisse des cotisants réguliers et la suscitation de coordinations hors syndicats obligent les centrales à porter leur effort sur le local et sur le marché de l'emploi (le «terrain »). C'est ainsi que syndicalisme de proximité cumule dans ses valeurs de sens une quadruple nécessité : accompagner le mouvement de décentralisation, s'intégrer au discours syndical européen, coller aux mutations du travail et du salariat et - pourquoi pas ? - s'inscrire en réaction contre les craintes suscitées par la mondialisation et le rentabilisme. Ce n'est pas sans raison que $M$. Rocard reconnait que «les services de proximité répondent à un besoin croissant des sociétés contemporaines $»^{13}$. Dans le domaine sociopolitique, il n'est aucun stéréotype qui ne corresponde aux exigences complexes d'une époque. 